\title{
Alternative route to airway relaxation
}

Promoting airway smooth muscle cell (ASMC) relaxation is an established therapeutic strategy for alleviating pulmonary resistance in asthma. However, current approaches, which involve the inhalation of $\beta_{2}$-adrenoceptor agonists, do not adequately control symptoms in all patients and are associated with side effects. Writing in Science Translational Medicine, Yin and colleagues now report the identification of an agonist of transgelin 2 (TG2; also known as TAGLN2), which induced ASMC relaxation and inhibited pulmonary resistance in mouse models of asthma.

The aetiology and mechanism of ASMC contraction in asthma are not well understood. Yin and colleagues therefore set out to investigate the potential role of metallothionein 2 (MT2), a protein expressed in mammalian airway epithelial cells and smooth muscle cells. MT2 has been shown to protect against pulmonary oxidative stress during endotoxaemia, but has not previously been studied in asthma.

First, the authors found that MT2 protein expression was more than $50 \%$ lower in asthmatic lung tissue of ovalbumin (OVA)-induced asthmatic rats compared with control animals. In addition, when challenged with OVA, pulmonary resistance was twofold higher in Mt2-knockout compared with wild-type asthmatic mice.

Conversely, injection of recombinant MT2 into OVA-induced asthmatic rats through an external jugular vein rapidly reduced pulmonary resistance, to a greater extent than that achieved with the $\beta_{2}$-agonist terbutaline. MT2 also significantly reduced the isometric tension of rat tracheal spirals by more than $60 \%$, and was more effective than terbutaline. Furthermore, MT2 had a greater effect than terbutaline on the relaxation of rat-derived primary ASMCs that had been challenged with acetylcholine.

Mechanistic studies revealed that MT2 binds to TG2 (an actin-binding protein that relaxes the cellular cytoskeleton) on the membrane of ASMCs; this interaction was specifically required for MT2-induced ASMC relaxation. Protein array analysis of the intracellular effects of TG2 activation by MT2 in rat ASMCs revealed phosphorylation of ezrin (a structural protein involved in stabilizing membrane receptor complexes) and the dephosphorylation of myosin phosphatase target subunit 1 (MYPT1; a regulatory subunit of myosin phosphatase that regulates the interaction of actin and myosin).

Next, the authors performed molecular docking analyses for more than 6000 compounds from an in-house database based on the NMR structure for the binding site of the TG2 calponin homology domain. Optimization of hit compounds led to the identification of the TG2 agonist TSG12, which inhibited acetylcholine-induced contraction of rat ASMCs and reduced asthmatic pulmonary resistance in mice. TSG12 induced MYPT1 dephosphorylation (as seen with MT2) and was shown to inactivate the RHOA-ROCKMYPT1-MLC pathway, thereby causing ASMC relaxation.

TSG12 inhalation inhibited pulmonary resistance by more than $80 \%$ in asthmatic mice that had been challenged with house dust mite, and was more effective than terbutaline. TSG12 similarly inhibited pulmonary resistance in OVA-challenged asthmatic mice, to a greater extent than that achieved with the $\beta$-agonist isoproterenol. Importantly, TSG12 also reduced acetylcholine-induced contraction of human ASMCs in a dose-dependent manner, without signs of toxicity or desensitization.

Together, these results suggest that TG2 agonists may represent a promising novel approach for treating asthma, with potential clinical advantages over existing therapies. The authors plan to structurally optimize TSG12 to obtain novel analogues with improved metabolic stability and solubility for further drug development.

Sarah Crunkhorn

ORIGINAL ARTICLE Yin, L.-M. et al. Transgelin-2 as a therapeutic target for asthmatic pulmonary resistance. Sci. Transl Med. 10, eaam8604 (2018) 